\begin{tabular}{|c|c|}
\hline \multirow{3}{*}{ 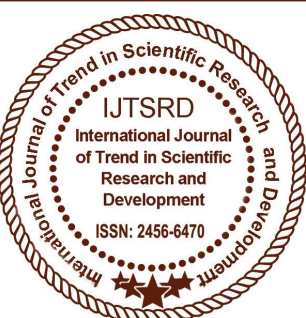 } & $\begin{array}{l}\text { International Journal of Trend in Scientific } \\
\text { Research and Development (IJTSRD) }\end{array}$ \\
\hline & International 0 \\
\hline & ISSN No: 2456 - 6470 | www.ijtsrd.com | Volume - 2 | Issue -4 \\
\hline
\end{tabular}

\title{
Cluster Based Resource Allocation for CoMP MU-MIMO 5G Network
}

\author{
Mr. M. Rubas ${ }^{1}$, Mr. G. Kannan ${ }^{2}$, Mrs. Dr. G. Gandhimathi ${ }^{3}$ \\ ${ }^{1}$ M.E Communication Systems, ${ }^{2}$ Assistant Professor, ${ }^{3}$ Professor \\ Department of ECE, Parisutham Institute of Technology \& Science, Thanjavur, Tamil Nadu, India
}

\begin{abstract}
Massive MIMO (multiple-input multiple-output) is considered as an heir of the multi-user MIMO technology and it has recently gained lots of attention from both academia and industry. In 5G environment, more users using the network at the same time, CoMP is used for MU-MIMO beamforming environment to provide network from BS. The transmit power, pilot training, and spatial transmission resources need to be allocated properly to the users to achieve the highest possible performance. This is called resource allocation and can be formulated as design utility optimization problems. Identifying non-disjoint clusters is an important issue in clustering referred to as Overlapping Clustering. While traditional clustering methods ignore the possibility that an observation can be assigned to several groups and lead to $\mathrm{k}$ exhaustive and exclusive clusters representing the data, Overlapping Clustering methods offer a richer model for fitting existing structures in several applications requiring a nondisjoint partitioning. In this Thesis Develop Proper Clustering algorithm for CoMP network based on location and traffic load using Similarity-based Clustering and Model-based Overlapping Clustering. The Simulation results analysis the performance of $\mathrm{K}$ Means and Hierarchical Clustering.
\end{abstract}

Keywords: CoMP network, $M U-M I M O, B S$, SBK, $M O C$

\section{INTRODUCTION}

The Third Generation Partnership Project (3GPP) Long Term Evolution (LTE)-Advanced is envisaged as the fourth generation cellular standard, and is aligned with existing third generation deployments, e.g., Universal Mobile Telecommunications System (UMTS). The goals of LTE-Advanced are to improve the peak throughput by increasing the numbers of transmit and receive antennas. One of the key enabling technologies of LTE-Advanced is coordinated multipoint (CoMP) that targets to improve the cell-edge performance as well as overall network spectral efficiency through base stations (BSs) coordination. In this thesis, develop proper clustering algorithms for CoMP network based on location and traffic load that provides high QoS while is proposed. The proposed algorithm takes advantage of frequency, spatial and time diversities in the timevarying wireless channel to increase the CoMP network performance. The concept of "overlapping user grouping", aiming to increase the system capacity and at the same time ensure full user coverage. To improving user experience in future $5 \mathrm{G}$ cellular networks.

\section{5G NETWORKS}

$5 \mathrm{G}$ network is very fast and reliable. The concept of hand held devices is going to be revolutionized with the advent of 5G. Now all the services and applications are going to be accessed by single IP as telephony, gaming and many other multimedia applications. As it is not a new thing in market and there are millions of users all over the world who have experienced the wireless services wireless technology. It is not easy for them to shrink from using this new $5 \mathrm{G}$ network technology. There is only need to make it accessible so that a common man can easily afford the profitable packs offered by the companies so that $5 \mathrm{G}$ network could hold the authentic place. There is need to win the customer trust to build fair long term relation to make a reliable position in the 
telecommunication field. To complete with the preceding wireless technologies in the market $5 \mathrm{G}$ network has to tender something reliable something more pioneering. All the features like telephony, camera, mp3 player, are coming in new mobile phone models. $4 \mathrm{G}$ is providing all these utility in mobile phone. By seeing the features of $4 \mathrm{G}$ one can gets a rough idea about what $5 \mathrm{G}$ Networks could offer. There is messenger, photo gallery, and multimedia applications that are also going to be the part of $5 \mathrm{G}$. There would be no difference between a $\mathrm{PC}$ and a mobile phone rather both would act vice versa.

\section{SYSTEM DESCRIPTION}

The main goal of the work is to develop Clustering algorithms for CoMP LTE-Advanced network that provides high QoS. The resource allocation approach should be able to take advantage of diversities offered in multiuser wireless networks, specifically in frequency, spatial and time domains. To develop Proper Clustering algorithms for CoMP network based on location and traffic load. The overlapping clustering model that we present here is a generalization of the SBK model.

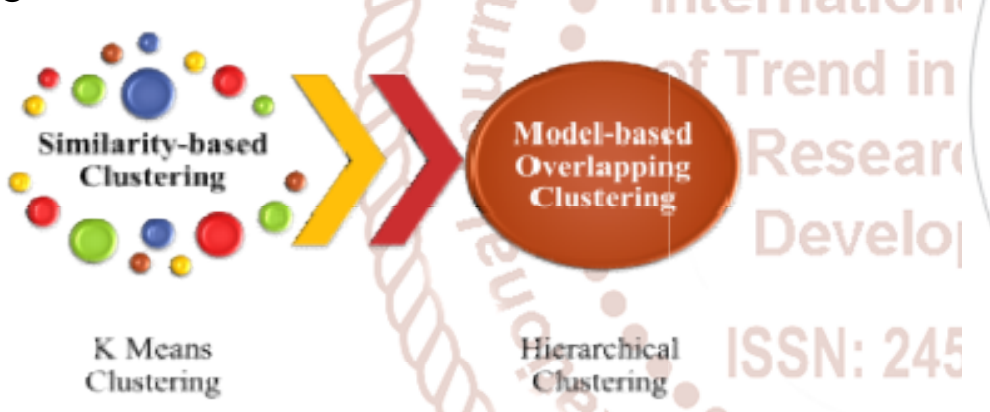

Fig 1: Proposed System

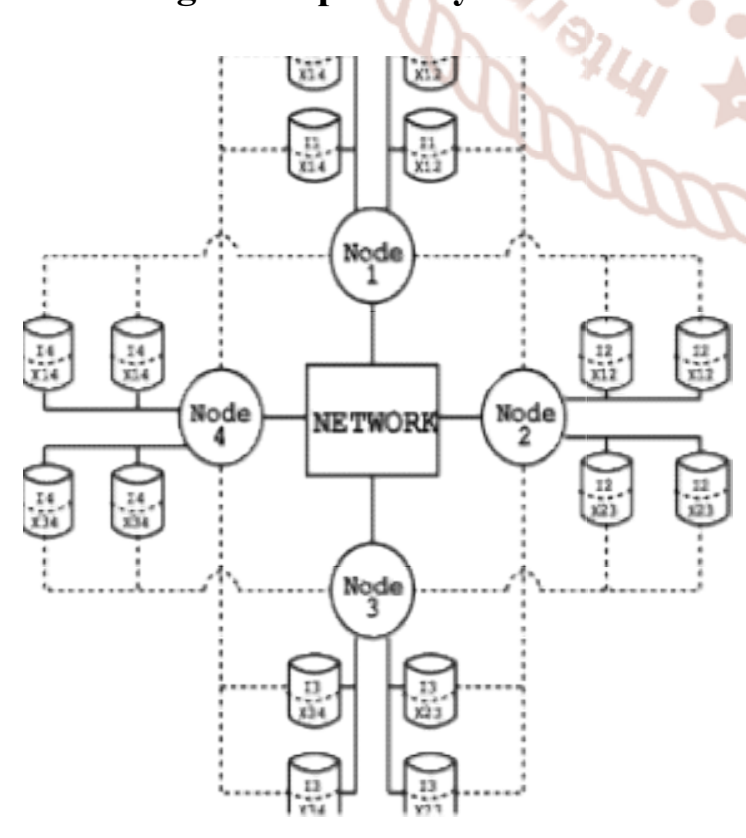

Fig 2: Block Diagram
To make the number of steps to construct the example as few as possible, the configuration is kept simple, rather than realistic. The example might represent the integration of two clusters created by two separate organizations. For a more realistic scenario, see Clustering: Planning how to configure cluster transmission queues.

Follow the steps to construct the clusters. The clusters are used in the following examples of isolating the message traffic from the client application to the server application.

The instructions add a couple of extra queue managers so that each cluster has two repositories. The gateway queue manager is not used as a repository for performance reasons.

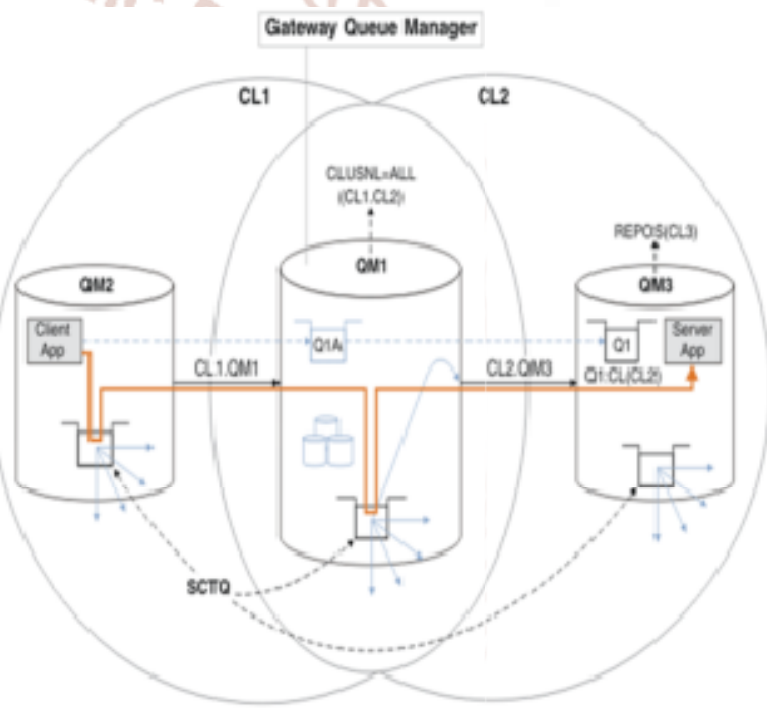

Fig 3: System Model

Given set of $\mathrm{n}$ data points, each point being a vector in $\mathrm{Rd}$, let them be represented by a $n^{*} d$ observation matrix X, such that row Xi denotes the ith data point and $\mathrm{Xij}$ represents its $\mathrm{jth}$ feature. Fitting a mixture model to $X$ is equivalent to assuming that each data point $\mathrm{Xi}$ is drawn independently from a probability density.

Let $\mathrm{Z}$ be a $n^{*} \mathrm{k}$ boolean matrix such that $\mathrm{Zij}$ is 1 if the $\mathrm{jth}$ component density was selected to generate $\mathrm{Xi}$, and 0 otherwise. In mixture model estimation, since each point $\mathrm{Xi}$ is assumed to be generated from only one underlying mixture component, every row $\mathrm{Zi}$ is a $\mathrm{k}$-dimensional boolean vector constrained to have 1 in only one column and 0 everywhere else. Let zi be a random variable corresponding to the index of the 1 in each row $\mathrm{Zi}$ : every $\mathrm{zi}$ is therefore a multinomial 
random variable, since it can take one of $\mathrm{k}$ discrete values. If the matrix $Z$ is known, one can directly estimate the parameters of the most likely model explaining the data by maximizing the complete loglikelihood of the observed data.

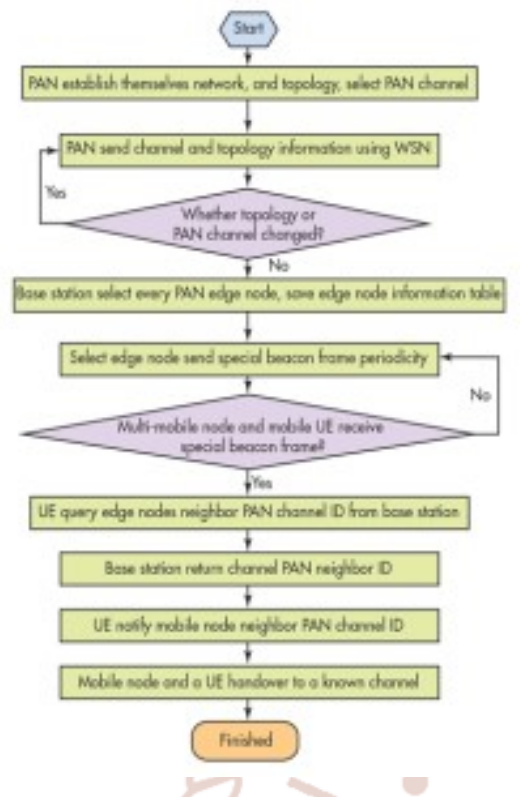

Fig 4: Flow Chart
The SBK model assumes that $\mathrm{M}$ and $\mathrm{A}$ are independent so that $\mathrm{P}(\mathrm{M}, \mathrm{A})=\mathrm{P}(\mathrm{M}) \mathrm{P}(\mathrm{A})$ and that Xij's are conditionally independent given $\mathrm{Mi}$ and $\mathrm{Aj}$. The SBK model minimizes the squared loss between $X$ and MA, and their proposed algorithms is not applicable for estimating the optimal $\mathrm{M}$ and $\mathrm{A}$ corresponding to other loss functions.

We show that the basic SBK model for overlapping clustering can be (more simply) understood as an extension of the mixture modeling with Gaussian density functions. We extend the basic SBK model to work with any regular exponential family. Using a connection between exponential families and Bregman divergences. We outline an alternating minimization algorithm for the general model that monotonically improves the objective function for overlapping models for any regular exponential family distribution. We present empirical evidence that the proposed overlapping clustering model works better than some alternative approaches to overlapping clustering.

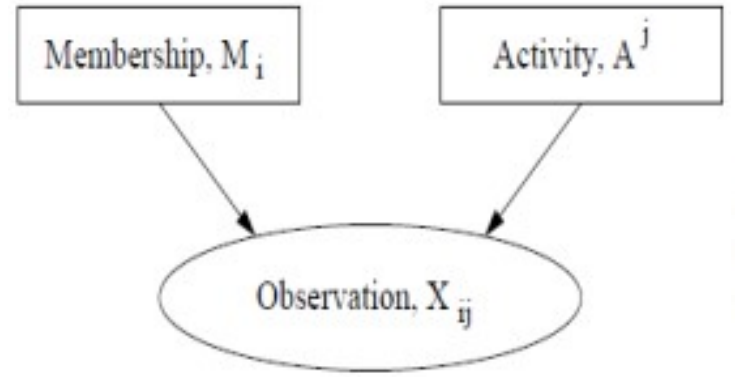

Fig 5: Similarity-based Clustering

In MOC, we generalize the SBK model to work with a broad class of probability distributions, instead of just Gaussians, and propose an alternate minimization algorithm for the general model.

The most important difference between MOC and the mixture model is that we remove the multinomial constraint on the matrix $Z$, so that it can now be an arbitrary boolean matrix. To distinguish from the constrained matrix $Z$, we denote this unconstrained boolean matrix as the membership matrix M. Every point $\mathrm{Xi}$ now has a corresponding k-dimensional boolean membership vector $\mathrm{Mi}$ : the $\mathrm{h}$ th component Mh i of this membership vector is a Bernoulli random variable indicating whether $\mathrm{Xi}$ belongs to the $\mathrm{h}$ th cluster. The membership vector Mi for the point $\mathrm{Xi}$ effectively encodes $2 \mathrm{k}$ configurations, starting from [00:: : : 4 , indicating that $\mathrm{Xi}$ does not belong to any cluster, to $[11::: 1 \Phi$, indicating that Xi belongs to all $\mathrm{k}$ clusters. So, a vector Mi with multiple 1's directly encodes the fact that the point Xi belongs to multiple clusters.

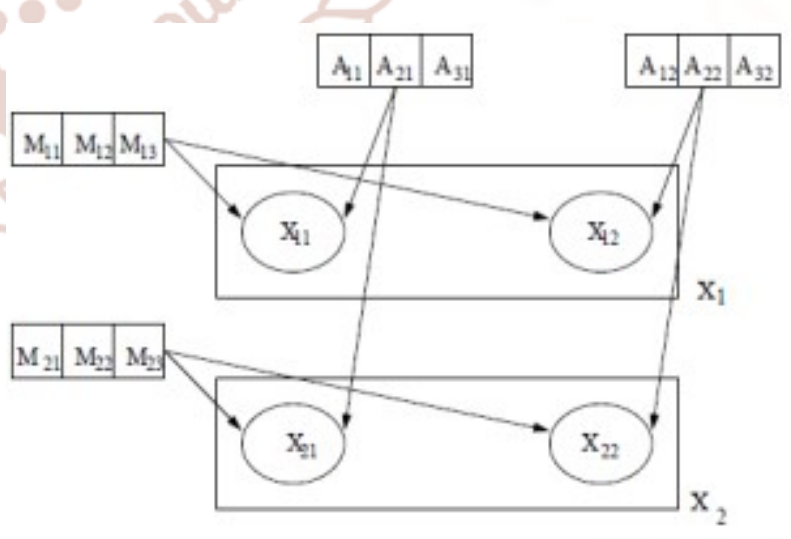

Fig 6: Model-based Overlapping Clustering

Such a procedure will of course depend on the order in which clusters are considered to be turned "on". In particular, the choice of the first cluster to be turned "on" will partly determine which other clusters will 
get turned "on". The permutation dependency of the problem is somewhat similar in flavor to that of payoff computation in a co-operative game. If $h$ players are already in cooperation, the value-add of the $(\mathrm{h}+1)$ th partner will depend on the permutation following which the first $\mathrm{h}$ were chosen. In order to design a fair pay-off strategy, one computes the average value-add of a player, better known as Shapley value, over all permutations of forming cooperations. The other entries of the permutation are obtained greedily on the fly. Since dynamicM runs $\mathrm{k}$ threads to achieve partial permutation independence, the best membership vector over all the threads is selected at the end. The algorithm has a worst case running time of $\mathrm{O}(\mathrm{k} 3)$ and is capable of running with any distance function.

\section{SYSTEM ANALYSIS}

$\mathrm{K}$ means is an iterative clustering algorithm that aims to find local maxima in each iteration. This algorithm works in these 6 steps:

1) Specify the desired number of clusters $K$ : Let us choose $\mathrm{k}=2$ for these 5 data points in 2-D space.

2) Randomly assign each data point to a cluster: Let's assign three points in cluster 1 shown using red color and two points in cluster 2 shown using grey color.

3) Compute cluster centroids: The centroid of data points in the red cluster is shown using red cross and those in grey cluster using grey cross.

4) Re-assign each point to the closest cluster centroid: Note that only the data point at the bottom is assigned to the red cluster even though its closer to the centroid of grey cluster. Thus, we assign that data point into grey cluster.

5) Re-compute cluster centroids: Now, re-computing the centroids for both the clusters.

6) Repeat steps 4 and 5 until no improvements are possible: Similarly, we'll repeat the 4th and 5th steps until we'll reach global optima. When there will be no further switching of data points between two clusters for two successive repeats. It will mark the termination of the algorithm if not explicitly mentioned.

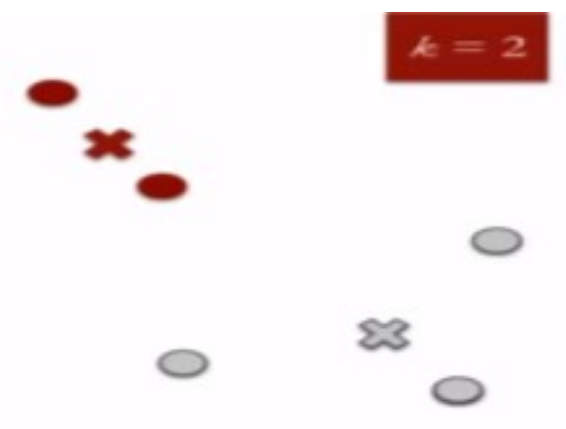

Fig 7: K Means Clustering

Hierarchical clustering, as the name suggests is an algorithm that builds hierarchy of clusters. This algorithm starts with all the data points assigned to a cluster of their own. Then two nearest clusters are merged into the same cluster. In the end, this algorithm terminates when there is only a single cluster left. The results of hierarchical clustering can be shown using dendrogram.

At the bottom, we start with 25 data points, each assigned to separate clusters. Two closest clusters are then merged till we have just one cluster at the top. The height in the dendrogram at which two clusters are merged represents the distance between two clusters in the data space.

The decision of the number of clusters that can best depict different groups can be chosen by observing the dendrogram. The best choice of the no. of clusters is the no. of vertical lines in the dendrogram cut by a horizontal line that can transverse the maximum distance vertically without intersecting a cluster.

In the above example, the best choice of number of clusters will be 4 as the red horizontal line in the dendrogram below covers maximum vertical distance AB.

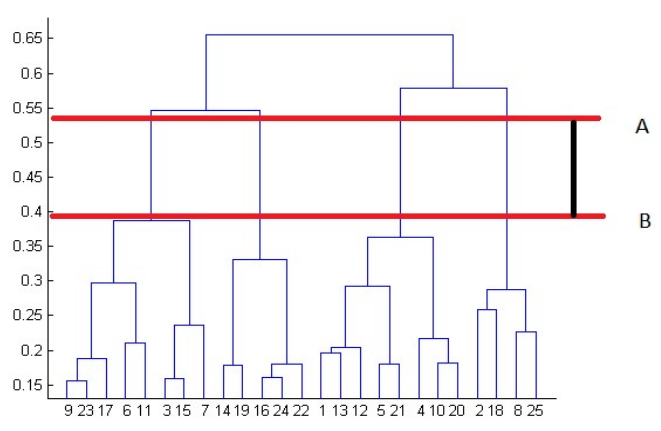

Fig 8: Hierarchical Clustering 
Two important things that you should know about hierarchical clustering are:

This algorithm has been implemented above using bottom up approach. It is also possible to follow top-down approach starting with all data points assigned in the same cluster and recursively performing splits till each data point is assigned a separate cluster.

$>$ The decision of merging two clusters is taken on the basis of closeness of these clusters. There are multiple metrics for deciding the closeness of two clusters :

-Euclidean distance: $\|\mathrm{a}-\mathrm{b}\| 2=\sqrt{ }(\Sigma($ ai-bi $))$

-Squared Euclidean distance: $\|\mathrm{a}-\mathrm{b}\| 22=\Sigma((\mathrm{ai}-\quad \mathrm{bi}) 2)$

-Manhattan distance: ||$a-b|| 1=\Sigma|a i-b i|$

-Maximum distance: ||a-b||INFINITY = maxi|ai-bi|

-Mahalanobis distance: $\sqrt{ }((a-b) T$ S-1 (-b)) \{where, $s$ : covariance matrix

The performance of the proposed clustering algorithm is carried out through simulation using MATLAB simulation environment. The network model and the proposed clustering and resource allocation algorithm is developed and evaluated through mathematical analysis.

\section{RESULTS}

In this thesis we proposed Clustering algorithms such as Similarity-based Clustering and Model-based Overlapping Clustering and their performance with single user and multiple user, with various diversities are analyzed under following four situation.

\section{K Means Clustering}

2. Hierarchical Clustering

3. UPGMA (Unweighted Pair Group Method with Arithmetic mean)

4. WPGMA (Weighted Pair Group Method with Arithmetic mean)

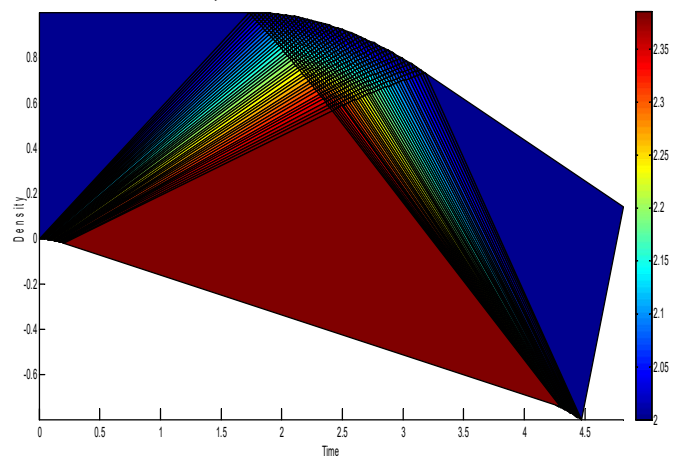

Fig 9: Cluster based Resource allocation

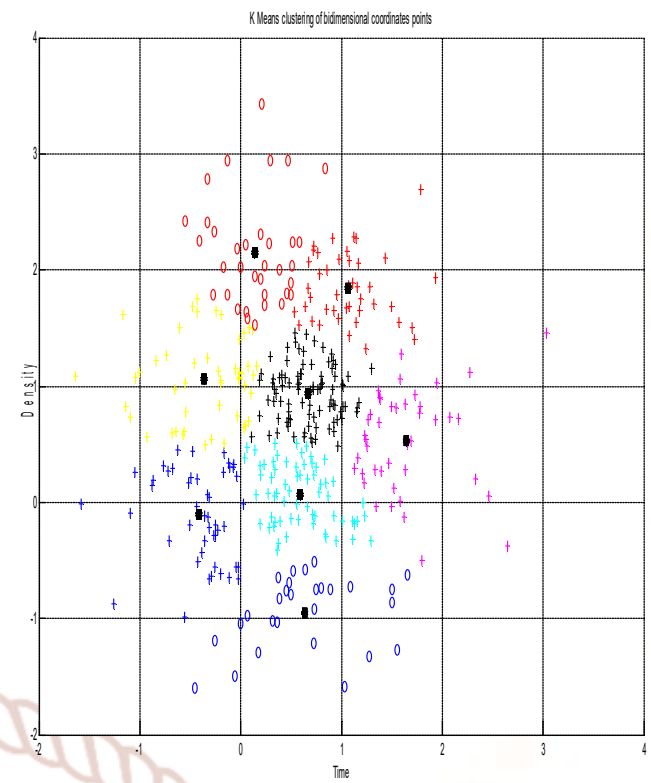

Fig 10: K Means clustering of bidimensional coordinates points

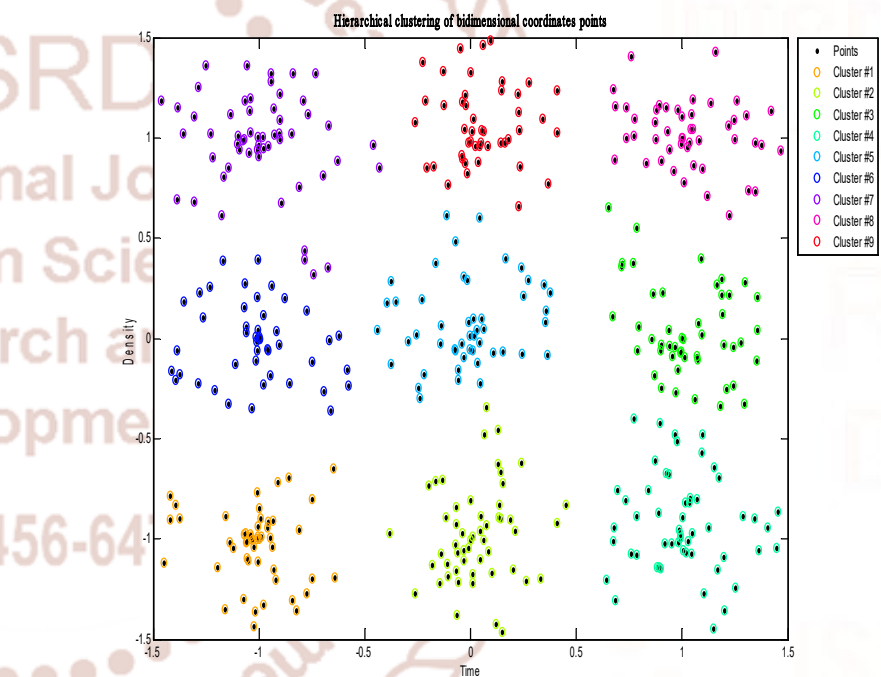

Fig 11: Hierarchical clustering of bidimensional coordinates points

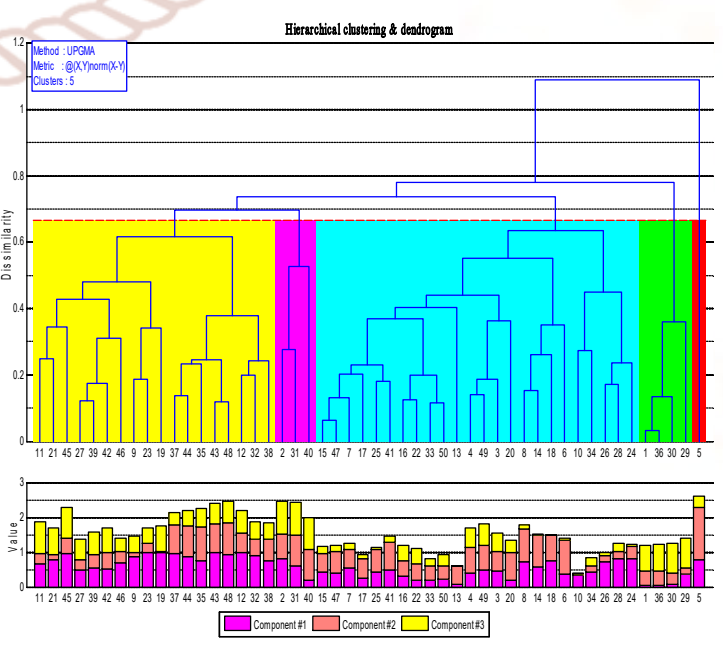

Fig 12: Dendrogram for Hierarchical clustering UPGMA method 


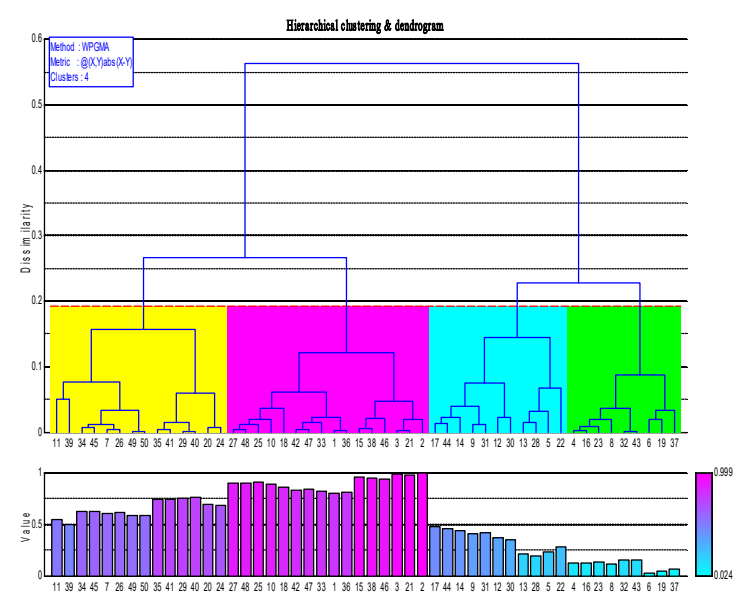

Fig 13: Dendrogram for Hierarchical clustering WPGMA method

In this research work we developed Clustering algorithms for CoMP network that can provide high QoS. The proposed algorithm has been proven to provide a significant improved performance for CoMP LTE-Advanced network and can be extended to future $5 \mathrm{G}$ network.

\section{CONCLUSION}

In wireless systems, interference is a major factor that limits the total network capacity. In this work, the allocation of system bandwidth and power among users in the network are coordinated such that the interference generated to other cells is minimized. This is also known as inter-cell interference coordination (ICIC), which is able to increase the overall network throughput.

In this thesis Clustering allows coordinate their transmission while the clusters compete with one another to reduce a per cluster cost based on their energy consumption and time load due to their traffic. Our proposed clustering method uses information on both the locations of BSs and their capability of handling the traffic and dynamically forms the clusters in order to improve the overall performance.

The broad generative model for overlapping clustering, MOC, based on generalizing the SBK model presented in. It has also provided a generic alternating minimization algorithm for efficiently and effectively fitting this model to empirical data. Finally, we have presented experimental results on both artificial data and real newsgroup and movie data, which demonstrate the generality and effectiveness of our approach. In particular, we have shown that the approach produces more accurate overlapping clusters than an alternative "naive" method based on thresholding the results of a traditional mixture model.

\section{REFERENCES}

1) Abdelnasser.A, E. Hossain, and D. I. Kim, "Clustering and resource allocation for dense femtocells in a two-tier cellular OFDMA network," IEEE Trans. Wireless Commun., vol. 13, no. 3, pp. 1628-1641, Mar.2014.

2) Baracca.P, F. Boccardi, and N. Benvenuto, "A dynamic clustering algorithm for downlink CoMP systems with multiple antenna UEs," EURASIP Journal on Wireless Communications and Networking, vol.2014, no. 1, 2014.

3) G. K. Yong, S. C., Jaekwon, K., Won, Y. Y. and Chung, MIMO-OFDM Wireless Communications with MATLAB. 2010. R. W. Peters, Steven W and Heath, "Cooperative algorithms for MIMO interference channels," Veh. Technol. IEEE Trans., vol. 60, pp. 206-218, 2011.

4) A. Mahmud, K. A. Hamdi, and N. Ramli, "Performance of fractional frequency reuse with comp at the cell-edge," 2014 Ieee Reg. 10 Symp., pp. 93-98, Apr. 2014.

5) J. P. Perez, F. Riera-Palou, and G. Femenias, "Combining fractional frequency reuse with coordinated multipoint transmission in MIMOOFDMA networks," 2013 IFIP Wirel. Days, pp. 1-8, Nov. 2013.

6) J. Li, H. Zhang, X. Xu, X. Tao, T. Svensson, C. Botella, and B. Liu, "A Novel Frequency Reuse Scheme for Coordinated Multi-Point Transmission," 2010 IEEE 71st Veh. Technol. Conf., pp. 1-5, 2010.

7) J. Hwang, S. M. Yu, S.-L. Kim, and R. Jantti, “On the Frequency Allocation for Coordinated MultiPoint Joint Transmission," 2012 IEEE 75th Veh.Technol. Conf. (VTC Spring), vol. 1, pp. 1-5, May 2012.

8) K. R. Han, Zhu and Liu, Resource allocation for wireless networks. Cambridge university press, 2008 .

9) G. Tychogiorgos and K. K. Leung, "Optimizationbased resource allocation in communication networks," Comput. Networks, vol. 66, pp. 32-45, Jun. 2014. 
10) D. Charilas, O. Markaki, D. Nikitopoulos, and M. Theologou, "Packet-switched network selection with the highest QoS in 4G networks," COMPUT NETW, vol. 52, no. 1, pp. 248-258, 2008.

11) L. Sun, Shaohui and Gao, Qiubin and Peng, Ying and Wang, Yingmin and Song, "Interference management through CoMP in 3GPP LTEadvance networks," Wirel. Commun. IEEE, vol. 20, no. 1, pp. 59-66, 2013.

12) M. Sawahaschi, Y. Kishiyama, A. Morimoto, D. Nishikawa, and M. Tanno, "Coordinated Multipoint Transmission/Reception Techniques for LTE- Advanced," IEEE Wirel. Commun., vol. 17, no. 3, pp. 26-34, 2010.

13) C. Yang, S. Han, X. Hou, and A. F. Molish, "How do we design CoMP to achieve Its Promised Potential?," IEEE Wirel. Commun., vol. 20, no. 1, pp.67-74, 2013.

14) G. P. Marsch, Patrick and Fettweis, Coordinated Multi-Point in Mobile Communications: from theory to practice. Cambridge University Press, 2011.

15) D. Lee, H. Seo, L. G. Electronics, B. Clerckx, S. Electronics, E. Hardouin, O. Labs, D. Mazzarese, and H. Technologies, "Coordinated Multipoint Transmission and Reception in LTE-Advanced : Deployment Scenarios and Operational Challenges," Commun. Mag. IEEE, vol. 50, no. 2, pp. 148-155, 2012.

16) D. Gesbert, S. Hanly, H. Huang, S. Shamai Shitz, O. Simeone, and W. Yu, "Multi-cell MIMO cooperative networks: A new look at interference," Sel. Areas Commun. IEEE J., vol. 28, no. 9, pp. 1380-1408, 2010.

17) Q. Zhang, C. Yang, and A. F. Molisch, "Cooperative downlink transmission mode selection under limited-capacity backhaul," 2012 IEEE Wirel. Commun. Netw. Conf., pp. 10821087, Apr. 2012.

18) Q. Zhang, C. Yang, and A. F. Molisch, "Downlink Base Station Cooperative Transmission," IEEE Trans. Wirel. Commun., vol. 12, no. 8, pp. 37463759, 2013.

19) P. Rost, "Robust and Efficient Multi-Cell Cooperation under Imperfect CSI and Limited Backhaul," IEEE Trans. Wirel. Commun., vol. 12, no. 4, pp.1910-1922, Apr. 2013.
20) C. Choi, L. Scalia, T. Biermann, and S. Mizuta, "Coordinated multipoint multiuser-MIMO transmissions over backhaul-constrained mobile access networks," 2011 IEEE 22nd Int. Symp. Pers. Indoor Mob. Radio Commun., pp. 13361340, Sep. 2011.

21) A. Zhang, Qian and Yang, Chenyang and Molisch, "Downlink Base Station Cooperative Transmission Under Limited-Capacity Backhaul," IEEE Trans. Wirel. Commun., vol. 12, no. 8, pp. 3746-3759, 2013.

22) T. Biermann, L. Scalia, C. Choi, H. Karl, and W. Kellerer, "CoMP clustering and backhaul limitations in cooperative cellular mobile access networks," Pervasive Mob. Comput., vol. 8, no. 5, pp. 662-681, Oct. 2012.

23) D. Hossain, Ekram and Le, Long Bao and Niyato, Radio resource management in multi-tier cellular wireless networks. John Wiley and Sons 2013.

24) K. LIU, Y. LI, H. JI, and X. WU, "Spectrum efficiency sub-carrier and energy efficiency power allocation for downlink multi-user CoMP in mucell system," J. China Univ. Posts Telecommunvol.21, no. 3, pp. 29-34, Jun. 2014.

25) X. Chen, X. Xu, H. Li, X. Tao, T. Svensson, and C. Botella, "Improved resource allocation strategy in SU-CoMP network," J. China Univ. Posts Telecommun., vol. 18, no. 4, pp. 7-12, Aug. 2011.

26) W. Cui, K. Niu, N. Li, and W. Wu, "Decentralized beamforming design and power allocation for limited coordinated multi-cell network," J. China Univ. Posts Telecommun., vol. 20, no. 4, pp. 5258, Aug. 2013.

27) D. Choi, S. Member, D. Lee, and J. H. Lee, "Resource Allocation for CoMP With Multiuser MIMO-OFDMA," IEEE Trans. Veh. Technol., vol. 60, no. 9, pp. 4626-4632, 2011. 